\section{Redesign of Hybrid Adaptive/Robust Motion Control of Rigid-Link Electrically-Driven Robot Manipulators}

\author{
Chun-Yi Su and Yury Stepanenko
}

\begin{abstract}
In a recent work of [16], a hybrid adaptive controller for rigid-link electrically-driven robot manipulators was proposed. Semiglobal asymptotic stability of the controller was established in the Lyapunov sense. However, there are two limitations in it. One is that the controller requires the joint velocity measurements, that with the required accuracy can be difficult to realize in practical applications. The other one assumes the boundedness of estimated inertia parameters of the manipulator in order to reduce the computation complexity. In this paper, we propose a modification of the hybrid adaptive controller, which eliminate the above-mentioned limitations. Hence the range of applicability of the method in [16] can be greatly broadened. The capabilities of the proposed control strategies are illustrated through computer simulation.
\end{abstract}

Index Terms-Actuators, robust control, adaptive control, robotic manipulators.

\section{INTRODUCTION}

Recently the problem of controlling the motion of rigid-link electrically-driven manipulators has received considerable attention, and many different approaches to this problem have been suggested [1]-[16]. Research in which controllers are designed with the capability to compensate for uncertainty in the manipulator/actuator system includes work on robust control schemes [6]-[9], adaptive schemes [10]-[13], and hybrid schemes [14]-[16]. It should be mentioned that these controllers usually require velocity measurements, that with the required accuracy can be difficult to realize in practical applications since joint measurements are typically either contaminated with noise or not available at all [17]. An additional observation is that derivation of these robust and adaptive schemes typically requires the calculation of very complex quantities, such as the time-derivative of the manipulator regressor matrix or upper bounds on the derivatives of the "embedded controls," which can make implementation of these strategies difficult and computationally expensive.

The schemes proposed in [15] and [16] also suffer the above mentioned draw backs. To avoid the derivative computation of the regressor matrix, a simplified algorithm was proposed in [16]. However, such an algorithm is only valid under the assumption that estimated inertia parameters of the manipulator are bounded. In this paper, we redesign the control scheme presented in [16] in an effort to eliminate the two limitations, these being the measurements of velocities and bounded assumption of estimated inertia parameters. It is shown that the proposed controller guarantees semi-global asymptotic link position tracking while also ensuring all signals remain bounded during closed-loop operation. Along the same line, a novel adaptive control algorithm [18] was also proposed to avoid

Manuscript received February 13, 1997; revised March 4, 1998. This paper was supported by the Natural Science and Engineering Research Council of Canada, the Institute for Robotic and Intelligent Systems (IRIS), and Precarn Associates Inc. This paper was recommended for publication by Associate Editor J. De Schutter and Editor A. Goldenberg upon evaluation of the reviewers' comments.

The authors are with Department of Mechanical Engineering, University of Victoria, Victoria, B.C. V8W 3P6, Canada.

Publisher Item Identifier S 1042-296X(98)04520-0. the velocity measurements. The main differences between the hybrid adaptive/robust and adaptive scheme were explained in [16].

\section{Design OF The Control LAW}

\section{A. Control Objective}

The dynamics for rigid-link electrically-driven manipulators are described by

$$
\begin{aligned}
(D(\boldsymbol{q})+J) \ddot{\boldsymbol{q}}+B(\boldsymbol{q}, \dot{\boldsymbol{q}}) \dot{\boldsymbol{q}}+G(\boldsymbol{q}) & =K_{N} \boldsymbol{I} \\
L \dot{\boldsymbol{I}}+R \boldsymbol{I}+K_{e} \dot{\boldsymbol{q}} & =\boldsymbol{u}
\end{aligned}
$$

where $q \in R^{n}$ is the vector of the joint position, $\boldsymbol{I} \in R^{n}$ is the vector of the armature currents and $\boldsymbol{u} \in R^{n}$ is the vector of the armature voltages; $D(\boldsymbol{q})$ is the manipulator mass-matrix, which is a symmetric positive definite matrix; $B(\boldsymbol{q}, \dot{\boldsymbol{q}}) \dot{\boldsymbol{q}}$ represents the centripetal and Coriolis force; $G(\boldsymbol{q})$ denotes the gravitational force; $J$ is the actuator inertia matrix; $L$ represents the actuator inductance matrix; $R$ is the actuator resistance matrix, $K_{e}$ is the matrix characterizing the voltage constant of the actuator and $K_{N}$ is the positive definite diagonal matrix which characterizes the electromechanical conversion between current and torque. While $D(\boldsymbol{q}), B(\boldsymbol{q}, \dot{\boldsymbol{q}}) \dot{\boldsymbol{q}}$ and $G(\boldsymbol{q})$ are nonlinear functions, $J, L, R, K_{e}$ and $K_{N}$ are positive definite constant diagonal matrices.

We attacks the same control objective as in [15] [16], i.e., for any given desired bounded trajectories $\boldsymbol{q}_{d}, \dot{\boldsymbol{q}}_{d}, \ddot{\boldsymbol{q}}_{d}$, and $\boldsymbol{q}_{d}^{(3)}$, with some or all of the manipulator and actuator parameters unknown, derive a controller for the actuator voltages $\boldsymbol{u}$ such that the manipulator position vector $q(t)$ tracks $q_{d}(t)$.

In accordance with the backstepping control strategy described by [19], the design procedure can be described as a two-step process. Firstly, the vector $I$ is regarded as a control variable for subsystem (1) and an embedded control input $\boldsymbol{I}_{d}$ is designed so that the tracking goal may be achieved. Secondly, $\boldsymbol{u}$ is designed such that $\boldsymbol{I}$ tracks $\boldsymbol{I}_{d}$. In turn, this allows $q(t)$ to track $q_{d}(t)$. In this paper (1) is called the manipulator subsystems and (2) the actuator subsystem.

\section{A. Adaptive Control for the Manipulator Subsystem}

Using the embedded armature current vector $I_{d}$, the model (1) can be rewritten as

$$
(D(\boldsymbol{q})+J) \ddot{\boldsymbol{q}}+B(\boldsymbol{q}, \dot{\boldsymbol{q}}) \dot{\boldsymbol{q}}+G(\boldsymbol{q})=K_{N} \boldsymbol{I}_{d}+K_{N} \tilde{\boldsymbol{I}}
$$

where $\tilde{\boldsymbol{I}} \triangleq \boldsymbol{I}-\boldsymbol{I}_{d}$ represents a current perturbating to the rigid-link dynamics. The system (3) can be viewed as a rigid model system with an input disturbance $K_{N} \tilde{\boldsymbol{I}}$, controlled by $K_{N} \boldsymbol{I}_{d}$.

Based on the parameterization technique [14], the nonlinear terms $D, B$, and $G$ in (1) can be expressed as

$$
\begin{gathered}
(D(\boldsymbol{q})+J) \ddot{\boldsymbol{q}}_{d}+B\left(\boldsymbol{q}, \dot{\boldsymbol{q}}_{d}\right) \dot{\boldsymbol{q}}_{d}+G(\boldsymbol{q}) \\
\quad=\Phi_{a}\left(\boldsymbol{q}, \dot{\boldsymbol{q}}_{d}, \ddot{\boldsymbol{q}}_{d}\right) \alpha_{a}
\end{gathered}
$$

where the term, $\Phi_{a}\left(\boldsymbol{q}, \dot{\boldsymbol{q}}_{d}, \ddot{\boldsymbol{q}}_{d}\right) \in R^{n \times(n \times m)}$, is the augmented regressor matrix independent of the dynamic parameters; the term, $\alpha_{a}^{T}$ is a corresponding augmented inertia parameter vector. Then, 
one has

$$
K_{N}^{-1} \Phi_{a} \alpha_{a}=\Phi_{a} K_{N a}^{-1} \alpha_{a}=\Phi_{a} \alpha_{a k}
$$

where $K_{N a} \triangleq \operatorname{diag}\left[k_{N i} I_{m}\right]$ and $\alpha_{a k}^{T} \triangleq K_{N a}^{-1} \alpha_{a}$.

We suppose that in the right hand side of (5) only the parameter vector $\alpha_{a k}$ is uncertain.' The desired $\boldsymbol{I}_{d}$ is then synthesized by

$$
\begin{aligned}
\boldsymbol{I}_{d} & =\Phi_{a}\left(\boldsymbol{q}, \dot{\boldsymbol{q}}_{d}, \ddot{\boldsymbol{q}}_{d}\right) \hat{\alpha}_{a k}-\gamma^{2} \Gamma(\boldsymbol{w}+\kappa \tilde{\boldsymbol{q}}) \\
\boldsymbol{w} & =\overline{\boldsymbol{w}}+\gamma^{2} \tilde{\boldsymbol{q}} \\
\dot{\overline{\boldsymbol{w}}} & =-2 \gamma \overline{\boldsymbol{w}}-2 \gamma^{3} \tilde{\boldsymbol{q}} \\
\dot{\hat{\alpha}}_{a k} & =\operatorname{Proj}\left(\hat{\alpha}_{a k},-\sigma \Phi_{a}^{T} z\right), \quad \hat{\alpha}_{a k}(0) \in \Pi \\
\boldsymbol{z} & \triangleq \dot{\tilde{\boldsymbol{q}}}-\frac{1}{\gamma} \boldsymbol{w}+\frac{\kappa}{\gamma} \tilde{\boldsymbol{q}}
\end{aligned}
$$

where $\tilde{\boldsymbol{q}} \triangleq \boldsymbol{q}-\boldsymbol{q}_{d}$ is the joint tracking error; $\hat{\alpha}_{a k}$ is the estimate of $\alpha_{a k} ; \Gamma$ is an arbitrary positive definite constant diagonal matrix; $\gamma, \kappa$, and $\sigma$ are positive constants; $\boldsymbol{w}$ and $\overline{\boldsymbol{w}}$ are intermediate vectors; $\operatorname{Proj}(\cdot, \cdot)$ is a projection operator, which is constructed as follows.

Choose a set $\Pi=\left\{\alpha_{a k} \mid \theta_{i \min }<\alpha_{a k i}<\theta_{i \max } \forall i \in\{1, n \times m\}\right\}$ with $\theta_{i \min }$ and $\theta_{i \max }$ some known real numbers. In this case, the projection operator defined by

$$
\begin{aligned}
& \left\{\operatorname{Proj}\left(\hat{\alpha}_{a k},-\sigma \Phi_{a}^{T} z\right)\right\}_{i} \\
& \quad= \begin{cases}0 & \text { if } \hat{\alpha}_{a k i}=\theta_{i \max } \text { and } \sigma\left(\Phi_{a}^{T} z\right)_{i}<0 \\
-\sigma\left(\Phi_{a}^{T} z\right)_{i} & \text { if }\left[\theta_{i \min }<\hat{\alpha}_{a k i}<\theta_{i \max }\right] \\
& \text { or }\left[\hat{\alpha}_{a k i}=\theta_{i \max } \text { and } \sigma\left(\Phi_{a}^{T} z\right)_{i} \geq 0\right] \\
& \text { or }\left[\hat{\alpha}_{a k i}=\theta_{i \min } \text { and } \sigma\left(\Phi_{a}^{T} z\right)_{i} \leq 0\right] \\
0 & \text { if } \hat{\alpha}_{a k i}=\theta_{i \min } \text { and } \sigma\left(\Phi_{a}^{T} z\right)_{i}>0\end{cases}
\end{aligned}
$$

satisfies

1) $\hat{\alpha}_{a k}(t) \in \Pi$ if $\hat{\alpha}_{a k}(0) \in \Pi$;

2) $\|\operatorname{Proj}(p, y)\| \leq\|y\|$;

3) $-\left(p-p^{*}\right)^{T} \Lambda \operatorname{Proj}(p, y) \geq-\left(p-p^{*}\right)^{T} \Lambda y$, where $\Lambda$ is a positive definite symmetric matrix.

\section{Remarks:}

1) The choice of $\theta_{i \text { min }}$ and $\theta_{i \text { mam }}$ is only related to the bound range of the projection operator and such a range in this paper is not restricted as long as the estimated parameters are bounded (required for the stability proof); hence one can always choose suitable $\theta_{i \min }$ and $\theta_{i \mathrm{mam}}$, although such a choice may be conservative.

2) It can easily be shown that $\hat{\alpha}_{a k}$ does not involve link velocity measurements, though $\dot{\hat{\alpha}}_{a k}$ includes the signal $\dot{\boldsymbol{q}}$. Therefore, $\boldsymbol{I}_{d}$ only needs link position measurements. This fact will be used later to prove that the controller for the overall system will depend only on the measurements of $\boldsymbol{I}$ and $\boldsymbol{q}$.

3) The role of the projection operator is crucial. The boundedness of the estimated parameters $\hat{\alpha}_{a k}$ can be guaranteed; as will be clear from the theorem proof, it is this boundedness that makes it possible to prove the semi-global stability of the overall system.

\section{Hybrid Adaptive Control for the Actuator Subsystem}

We now turn to the development of a voltage input $\boldsymbol{u}$, which forces $\tilde{\boldsymbol{I}}$ to zero. However, as shown in [16], using the backstepping technique [19], we are required to calculate

$$
\dot{\boldsymbol{I}}_{d}=(d / d t)\left(\Phi_{a}\left(\boldsymbol{q}, \dot{\boldsymbol{q}}_{d}, \ddot{\boldsymbol{q}}_{d}\right) \hat{\alpha}_{a k}\right)-\gamma^{2} \Gamma(\dot{\boldsymbol{w}}+\kappa \dot{\tilde{\boldsymbol{q}}})
$$

where $(d / d t)\left(\Phi_{a} \hat{\alpha}_{a k}\right)=\dot{\Phi}_{a} \hat{\alpha}_{a k}+\Phi_{a} \dot{\hat{\alpha}}_{a k}$. Hence, the calculation of $\dot{\Phi}_{a}$ is involved. Also, the calculations of derivative $\dot{\boldsymbol{I}}_{d}$ require measurements of the velocity $\dot{\boldsymbol{q}}$. The challenge addressed here is to design the control input $\boldsymbol{u}$ without involving the computation of $\dot{\Phi}_{a}$ and the measurements of $\dot{\boldsymbol{q}}$. In order to do so, we divide the embedded signals $\boldsymbol{I}_{d}$ as

$$
\begin{aligned}
& \boldsymbol{I}_{d} \triangleq \boldsymbol{I}_{p}+\boldsymbol{I}_{c} \\
& \boldsymbol{I}_{p} \triangleq \gamma^{2} \Gamma\left(\gamma^{2} \tilde{\boldsymbol{q}}-\boldsymbol{w}\right) \\
& \boldsymbol{I}_{c} \triangleq \Phi_{a}\left(\boldsymbol{q}, \dot{\boldsymbol{q}}_{d}, \ddot{\boldsymbol{q}}_{d}\right) \hat{\alpha}_{a k}-\gamma^{2} \Gamma\left(\gamma^{2}+\kappa\right) \tilde{\boldsymbol{q}}
\end{aligned}
$$

and simply substitute

$$
\dot{\boldsymbol{I}}_{p}=\gamma^{2} \Gamma\left(\gamma^{2} \dot{\tilde{\boldsymbol{q}}}-\dot{\boldsymbol{w}}\right)=2 \gamma^{3} \Gamma \boldsymbol{w}
$$

for $\dot{\boldsymbol{I}}_{d}$. The effect of the signal $\boldsymbol{I}_{c}$ will be compensated in the actuator subsystem. We note that in (15) the relation $\dot{\boldsymbol{w}}=-2 \gamma \boldsymbol{w}+\gamma^{2} \dot{\tilde{\boldsymbol{q}}}$ has been used. So, no velocity $\dot{\boldsymbol{q}}$ is involved in (15).

Following [16], it is assumed that the electrical parameters $K_{N}, L, R$, and $K_{e}$ are all of uncertain values. However, there exist $L_{0}, R_{0}$, and $K_{e o}$, all known, such that

$$
\left\|L-L_{0}\right\| \leq \delta_{1} ;\left\|R-R_{0}\right\| \leq \delta_{2} ;\left\|K_{e}-K_{e o}\right\| \leq \delta_{3}
$$

With the above in mind, the adaptive robust control law, forcing $\tilde{\boldsymbol{I}}=0$, is then synthesized by

$$
\begin{aligned}
\boldsymbol{u}= & L_{0} \dot{\boldsymbol{I}}_{p}+R_{0} \boldsymbol{I}_{d}+K_{e o} \dot{\boldsymbol{q}}_{d} \\
& -\left(\hat{\delta}_{1}\left\|\dot{\boldsymbol{I}}_{p}\right\|+\hat{\delta}_{2}\left\|\boldsymbol{I}_{d}\right\|+\hat{\delta}_{3}\left\|\dot{\boldsymbol{q}}_{d}\right\|\right) \operatorname{sgn}(\tilde{\boldsymbol{I}}) \\
\dot{\hat{\delta}}_{1}= & \eta_{1}\left\|\dot{\boldsymbol{I}}_{p}\right\|\|\tilde{\boldsymbol{I}}\| \\
\dot{\hat{\delta}}_{2}= & \eta_{2}\left\|\boldsymbol{I}_{d}\right\|\|\tilde{\boldsymbol{I}}\| \\
\dot{\hat{\delta}}_{3}= & \eta_{3}\left\|\dot{\boldsymbol{q}}_{d}\right\|\|\tilde{\boldsymbol{I}}\|
\end{aligned}
$$

where $\boldsymbol{I}_{d}, \boldsymbol{I}_{p}$, and $\dot{\boldsymbol{I}}_{p}$ are defined in (6), (13), and (15), $\hat{\alpha}_{a k}$ is given by (9), $\eta_{i}(i=1,2,3)$ are constants which determine the rates of adaptations.

Remarks:

1) Thanks to the definition of $I_{p}$, the time derivative of $I_{p}$ does not involve the velocity measurements, which in turn implies no velocity measurements in the controller (17). Thus, the cascade control system only requires the measurements of $I$ and $q$.

2) It is clear from (17), the time-derivative of the manipulator regressor matrix or upper bounds on the derivatives of the embedded controls are not involved. Therefore, the difficulty encountered in [15], [16] is removed.

3) Using the adaptive method, a scheme without using the velocity measurements was also proposed in [18]. The difference between adaptive and hybrid adaptive/robust schemes was discussed in the remark 3) of [16] and the reader may refer to it.

4) Similar to [16], the control law (17) involves the discontinuous function and may result in chattering behavior. For a discussion on how to remedy this the reader may refer to the remarks 4) and 5) of [16].

\section{Stability Analysis}

The stability of the closed-loop system described by (1), (2), (6), and (17) is established in the following theorem.

Theorem: If the robust control voltages $\boldsymbol{u}$ given by (6) and (17) are applied to the manipulator (1-2), then all closed-loop signals are bounded and $\lim _{t \rightarrow \infty} \tilde{\boldsymbol{q}}=0$, provided $\gamma$ initially satisfies:

1) $\gamma \geq \max \{1, \kappa\}$

2) the matrices $P$ and $Q$ in (24) being positive-definite; 
3) see (21)

$$
\gamma \lambda_{q}>3\left\|B_{d}\right\|+\mu_{1}+2 \vartheta\left\|\boldsymbol{q}_{d}\right\|+2 \vartheta \sqrt{\frac{\lambda_{v 2}}{\lambda_{v 1}}}\left\|\boldsymbol{x}_{v}(0)\right\|
$$

where $\lambda_{q}$ is defined in (24); $\lambda_{v 1}, \lambda_{v 1}$, and $x_{v}$, are defined in (28) while

$$
\begin{aligned}
& \mu_{1} \triangleq \frac{\beta_{1}^{2}}{4 \lambda_{r}}, \quad \beta_{1}=\left(3\left\|K_{N}\right\|+\varsigma+\left\|K_{e}\right\|\right), \quad \text { and } \\
& \lambda_{r} \triangleq \inf \frac{\tilde{\boldsymbol{I}}^{T} R \tilde{\boldsymbol{I}}}{\|\tilde{\boldsymbol{I}}\|^{2}} .
\end{aligned}
$$

Proof: Some mathematical preparations are necessary before the proof of stability. Substituting (6) into (3), one obtains the joint position error equation

$$
\begin{aligned}
K_{N}^{-1}(D(\boldsymbol{q})+J) \ddot{\boldsymbol{q}} & \\
= & -\gamma^{2} \Gamma \boldsymbol{w}-\kappa \gamma^{2} \Gamma \tilde{\boldsymbol{q}}+\tilde{\boldsymbol{I}}-K_{N}^{-1} B(\boldsymbol{q}, \dot{\boldsymbol{q}}) \dot{\tilde{\boldsymbol{q}}} \\
& -K_{N}^{-1} B_{d} \dot{\tilde{\boldsymbol{q}}}+\Phi_{a} \tilde{\alpha}_{a k}
\end{aligned}
$$

where $\tilde{\alpha}_{a k} \triangleq \hat{\alpha}_{a k}-\alpha_{a k}$ and $B_{d} \dot{\tilde{\boldsymbol{q}}} \triangleq B\left(\boldsymbol{q}, \dot{\boldsymbol{q}}_{d}\right) \dot{\tilde{\boldsymbol{q}}}=B(\boldsymbol{q}, \dot{\boldsymbol{q}}) \dot{\boldsymbol{q}}_{d}-$ $B\left(\boldsymbol{q}, \dot{\boldsymbol{q}}_{d}\right) \dot{\boldsymbol{q}}_{d}$, therein, the property $B(\boldsymbol{q}, \dot{\boldsymbol{q}}) \dot{\boldsymbol{q}}_{d}=B\left(\boldsymbol{q}, \dot{\boldsymbol{q}}_{d}\right) \dot{\boldsymbol{q}}$ has been used. It can be shown that $B_{d}$ is a uniformly bounded matrix because $\dot{\boldsymbol{q}}_{d}$ is uniformly bounded.

Using a state vector $\boldsymbol{x}^{T} \triangleq\left[\dot{\tilde{\boldsymbol{q}}}^{T}, \boldsymbol{w}^{T}, \tilde{\boldsymbol{q}}^{T}\right]$, the dynamic equation (22) can be expressed in state space as

$$
\dot{\boldsymbol{x}}=-A \boldsymbol{x}+C\left(\tilde{\boldsymbol{I}}-K_{N}^{-1} B(\boldsymbol{q}, \dot{\boldsymbol{q}}) \dot{\tilde{\boldsymbol{q}}}-K_{N}^{-1} B_{d} \dot{\tilde{\boldsymbol{q}}}+\Phi_{a} \tilde{\alpha}_{a k}\right)
$$

where the detailed expressions for $A$ and $C$ are given in [16] and omitted here.

As shown in [16], by properly choosing a pair of positive definite matrices $P$ and $Q$, the relation $\frac{1}{2}\left(P A+A^{T} P\right)=Q$ can be established and they satisfy

$$
\lambda_{p}\|x\|^{2} \leq \boldsymbol{x}^{T} P \boldsymbol{x} \text { and } \gamma \lambda_{q}\|\boldsymbol{x}\|^{2} \leq \boldsymbol{x}^{T} Q \boldsymbol{x}
$$

where $\lambda_{p}$ and $\lambda_{q}$ denote the smallest eigenvalues of the matrices $P$ and $(1 / \gamma) Q$.

At this point, the closed-loop stability poof can be established by using a Lyapunov function candidate

$$
\begin{aligned}
V(t) & =L_{a}(t)+\bar{L}_{i}(t) \\
L_{a} & \triangleq \frac{1}{2} \boldsymbol{x}^{T} P \boldsymbol{x}+\frac{1}{2 \sigma} \tilde{\alpha}_{a k}^{T} K_{N a} \tilde{\alpha}_{a k}
\end{aligned}
$$

where $\boldsymbol{x}$ is defined in (23), $\tilde{\alpha}_{a k}=\hat{\alpha}_{a k}-\alpha_{a k}$, therein, $\alpha_{a k}$ is defined in (5) and $\hat{\alpha}_{a k}$ is its estimate; and

$$
\bar{L}_{i}(t) \triangleq \frac{1}{2} \tilde{\boldsymbol{I}}^{T} L \tilde{\boldsymbol{I}}+\frac{1}{2} \sum_{i=1}^{3}\left(\breve{\delta}_{i}-\hat{\delta}_{i}\right)^{2} / \eta_{i}
$$

where $\breve{\delta}_{1}=\delta_{1}, \breve{\delta}_{2}=\delta_{2}$, and $\breve{\delta}_{3}=\delta_{3}+\zeta, \delta_{i}(i=1,2,3)$ are defined in (16), $\zeta$ is defined in (33), and $\hat{\delta}_{i}$ are the estimates of $\breve{\delta}_{i}$. Given (25), one has

$$
\lambda_{v 1}\left\|\boldsymbol{x}_{v}\right\|^{2} \leq V \leq \lambda_{v 2}\left\|\boldsymbol{x}_{v}\right\|^{2}
$$

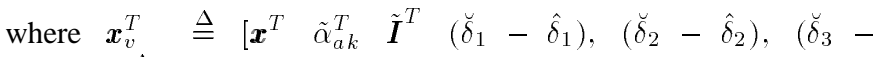
$\left.\left.\hat{\delta}_{3}\right)\right] ; \lambda_{v 1} \triangleq \frac{1}{2} \min \left\{\lambda_{\min }(P), \lambda_{\min }\left(K_{N a}\right) / \sigma, \lambda_{\min }(L), 1 / \eta_{i}(i=\right.$ $1,2,3)\}$ and $\lambda_{v 2} \triangleq \frac{1}{2} \max \left\{\lambda_{\max }(P), \lambda_{\max }\left(K_{N a}\right) / \sigma, \lambda_{\max }(L)\right.$, $\left.1 / \eta_{i}(i=1,2,3)\right\}$.

The time derivative of $L_{a}(t)$ along the trajectory of (23) is

$$
\begin{aligned}
\dot{L}_{a}= & -\boldsymbol{x}^{T} Q \boldsymbol{x}+\boldsymbol{x}^{T} P C \tilde{\boldsymbol{u}}+\boldsymbol{x}^{T} P C\left(\tilde{\boldsymbol{I}}-K_{N}^{-1} B(\boldsymbol{q}, \dot{\boldsymbol{q}}) \dot{\tilde{\boldsymbol{q}}}\right. \\
& \left.-K_{N}^{-1} B_{d} \dot{\tilde{\boldsymbol{q}}}+\Phi_{a} \tilde{\alpha}_{a k}\right)+\frac{1}{2} \boldsymbol{x}^{T} \dot{\boldsymbol{P}} \boldsymbol{x}+\frac{1}{\sigma} \dot{\tilde{\alpha}}_{a k}^{T} K_{N a} \tilde{\alpha}_{a k} .
\end{aligned}
$$

Following the derivations in [16], when $\gamma \geq \max \{1, \kappa\}$, one can obtain

$$
\begin{aligned}
\dot{L}_{a} \leq & -\left(\gamma \lambda_{q}-3\left\|B_{d}\right\|-2 \vartheta\|\dot{\boldsymbol{q}}\|\right)\|x\|^{2}+3 \alpha_{n}\|x\|\|\tilde{\boldsymbol{I}}\| \\
& +z^{T} K_{N} \Phi_{a} \tilde{\alpha}_{a k}+\frac{1}{\sigma} \dot{\tilde{\alpha}}_{a k}^{T} K_{N a} \tilde{\alpha}_{a k} \\
= & -\left(\gamma \lambda_{q}-3\left\|B_{d}\right\|-2 \vartheta\|\dot{\boldsymbol{q}}\|\right)\|x\|^{2}+3 \alpha_{n}\|x\|\|\tilde{\boldsymbol{I}}\| \\
& +z^{T} K_{N} \Phi_{a} \tilde{\alpha}_{a k} \\
& +\frac{1}{\sigma} \tilde{\alpha}_{a k}^{T} K_{N a} \operatorname{Proj}\left(\hat{\alpha}_{a k},-\sigma \Phi_{a}^{T} z\right) \\
\leq & -\left(\gamma \lambda_{q}-3\left\|B_{d}\right\|-2 \vartheta\|\dot{\boldsymbol{q}}\|\right)\|x\|^{2}+3 \alpha_{n}\|x\|\|\tilde{\boldsymbol{I}}\| \\
& +z^{T} \Phi_{a} \tilde{\alpha}_{a}-z^{T} \Phi_{a} K_{N a} \tilde{\alpha}_{a k} \\
= & -\left(\gamma \lambda_{q}-3\left\|B_{d}\right\|-2 \vartheta\|\dot{\boldsymbol{q}}\|\right)\|x\|^{2}+3 \alpha_{n}\|x\|\|\tilde{\boldsymbol{I}}\|
\end{aligned}
$$

where $\alpha_{n} \triangleq\left\|K_{N}\right\|$, and the identity $x^{T} P C K_{N}^{-1}=z^{T}$ and the property $(1 / \sigma) \tilde{\alpha}_{a k}^{T} K_{N a} \operatorname{Proj}\left(\hat{\alpha}_{a k},-\sigma \Phi_{a}^{T} z\right) \leq-\tilde{\alpha}_{a k}^{T} K_{N a} \Phi_{a}^{T} z$ have been used.

The time derivative of $\bar{L}_{i}(t)$ is evaluated along the trajectory (2) as

$$
\begin{aligned}
\dot{\bar{L}}_{i}= & -\tilde{\boldsymbol{I}}^{T}\left[L\left(\dot{\boldsymbol{I}}_{p}+\dot{\boldsymbol{I}}_{c}\right)+R \tilde{\boldsymbol{I}}+K_{e} \dot{\tilde{\boldsymbol{q}}}-\boldsymbol{u}+R \boldsymbol{I}_{d}+K_{e} \dot{\boldsymbol{q}}_{d}\right] \\
& +\sum_{i=1}^{3}\left(\breve{\delta}_{i}-\hat{\delta}_{i}\right)\left(-\dot{\hat{\delta}}_{i}\right) / \eta_{i} .
\end{aligned}
$$

When $\gamma \geq \max \{1, \kappa\}$, one can write

$$
-\tilde{\boldsymbol{I}}^{T} L \dot{\boldsymbol{I}}_{c}=-\tilde{\boldsymbol{I}}^{T} L\left(\dot{\Phi}_{a} \hat{\alpha}_{a k}+\Phi_{a} \dot{\hat{\alpha}}_{a k}-\gamma^{2} \Gamma\left(\gamma^{2}+\kappa\right) \dot{\tilde{\boldsymbol{q}}}\right) .
$$

Since $\dot{\boldsymbol{q}}_{d}, \ddot{\boldsymbol{q}}_{d}$ and $\boldsymbol{q}_{d}^{(3)}$ are uniformly bounded, one can write

$$
\left\|\Phi_{a}\right\| \leq \rho ; \quad\left\|\dot{\Phi}_{a}\right\| \leq \varrho\|\dot{\boldsymbol{q}}\| \leq \varrho\left(\|\dot{\tilde{\boldsymbol{q}}}\|+\left\|\dot{\boldsymbol{q}}_{d}\right\|\right)
$$

where $\rho$ and $\varrho$ are constants. Therefore, based on the property that $\left\|\operatorname{Proj}\left(\hat{\alpha}_{a k},-\sigma \Phi_{a}^{T} z\right)\right\| \leq \sigma\left\|\Phi_{a}^{T} z\right\|$, the (32) becomes

$$
\begin{aligned}
-\tilde{\boldsymbol{I}}^{T} L \dot{\boldsymbol{I}}_{c} \leq & \alpha_{l}\|\tilde{\boldsymbol{I}}\|\left(\left\|\dot{\Phi}_{a}\right\|\left\|\hat{\alpha}_{a k}\right\|+\left\|\Phi_{a}\right\|\left\|\dot{\hat{\alpha}}_{a k}\right\|\right. \\
& \left.+\gamma^{2} \Gamma\left(\gamma^{2}+\kappa\right)\|\dot{\tilde{\boldsymbol{q}}}\|\right) \\
\leq & \alpha_{l} \varrho\left\|\theta_{\max }\right\|\|\tilde{\boldsymbol{I}}\|\left(\|\dot{\tilde{\boldsymbol{q}}}\|+\left\|\dot{\boldsymbol{q}}_{d}\right\|\right) \\
& \left.+\alpha_{l}\|\tilde{\boldsymbol{I}}\|\left(\sigma \rho^{2} \| \boldsymbol{z}\right)\left\|+\gamma^{2} \Gamma\left(\gamma^{2}+\kappa\right)\right\| \dot{\tilde{\boldsymbol{q}}} \|\right) \\
\leq & \alpha_{l} \varrho\left\|\theta_{\max }\right\|\|\tilde{\boldsymbol{I}}\|\left(\|\boldsymbol{x}\|+\left\|\dot{\boldsymbol{q}}_{d}\right\|\right) \\
& +\alpha_{l}\|\tilde{\boldsymbol{I}}\|\left(3 \sigma \rho^{2}\|\boldsymbol{x}\|+\gamma^{2} \Gamma\left(\gamma^{2}+\kappa\right)\|\boldsymbol{x}\|\right) \\
= & \varsigma\|\tilde{\boldsymbol{I}}\|\|\boldsymbol{x}\|+\zeta\|\tilde{\boldsymbol{I}}\|\left\|\dot{\boldsymbol{q}}_{d}\right\|
\end{aligned}
$$

where $\varsigma \triangleq \alpha_{l}\left(\varrho\left\|\theta_{\max }\right\|+3 \sigma \rho^{2}+\gamma^{2} \Gamma\left(\gamma^{2}+\right.\right.$ $\kappa)), \zeta \triangleq \alpha_{l} \varrho\left\|\theta_{\max }\right\|, \alpha_{l} \triangleq\|L\|, \quad$ and $\left\|\hat{\alpha}_{a k}\right\| \leq$ $\left\|\theta_{\max }\right\|, \theta_{\max } \triangleq\left[\theta_{1 \max } \cdots \theta_{(n \times m) \max }\right]^{T}, \quad$ and $\theta_{i \max } \quad$ is defined in (11).

Substituting $\boldsymbol{u}$ in (31) by the control law (17) and noticing (16) and (33), one obtains

$$
\begin{aligned}
\dot{L}_{i} \leq & -\tilde{\boldsymbol{I}}^{T} R \tilde{\boldsymbol{I}}_{-} \tilde{\boldsymbol{I}}^{T} K_{e} \dot{\tilde{\boldsymbol{q}}}-\tilde{\boldsymbol{I}}^{T} L \dot{\boldsymbol{I}}_{c} \\
& +\left(\delta_{1}\left\|\dot{\boldsymbol{I}}_{p}\right\|\|\tilde{\boldsymbol{I}}\|+\delta_{2}\left\|\boldsymbol{I}_{d}\right\|\|\tilde{\boldsymbol{I}}\|+\delta_{3}\left\|\dot{\boldsymbol{q}}_{d}\right\|\|\tilde{\boldsymbol{I}}\|\right) \\
& -\left(\hat{\delta}_{1}\left\|\dot{\boldsymbol{I}}_{p}\right\|\|\tilde{\boldsymbol{I}}\|+\hat{\delta}_{2}\left\|\boldsymbol{I}_{d}\right\| \tilde{\boldsymbol{I}}\left\|+\hat{\delta}_{3}\right\| \dot{\boldsymbol{q}}_{d}\|\| \tilde{\boldsymbol{I}} \|\right) \\
& +\sum_{i=1}^{3}\left(\breve{\delta}_{i}-\hat{\delta}_{i}\right)\left(-\dot{\hat{\delta}}_{i}\right) / \eta_{i} \\
\leq & -\tilde{\boldsymbol{I}}^{T} R \tilde{\boldsymbol{I}}-\tilde{\boldsymbol{I}}^{T} K_{e} \dot{\tilde{\boldsymbol{q}}} \\
& +\left(\delta_{1}\left\|\dot{\boldsymbol{I}}_{p}\right\|\|\tilde{\boldsymbol{I}}\|+\delta_{2}\left\|\boldsymbol{I}_{d}\right\|\|\tilde{\boldsymbol{I}}\|+\delta_{3}\left\|\dot{\boldsymbol{q}}_{d}\right\|\|\tilde{\boldsymbol{I}}\|\right) \\
& +\varsigma\|\tilde{\boldsymbol{I}}\|\|\boldsymbol{x}\|+\zeta\left\|\tilde{\boldsymbol{I}}_{\|}\right\|\left\|\dot{\boldsymbol{q}}_{d}\right\| \\
& -\left(\hat{\delta}_{1}\left\|\dot{\boldsymbol{I}}_{m}\right\|\left\|\tilde{\boldsymbol{I}}_{\|}\right\|+\hat{\delta}_{2}\left\|\boldsymbol{I}_{d}\right\| \tilde{\boldsymbol{I}}\left\|+\hat{\delta}_{3}\right\| \dot{\boldsymbol{q}}_{d}\|\| \tilde{\boldsymbol{I}} \|\right) \\
& +\sum_{i=1}^{3}\left(\breve{\delta}_{i}-\breve{\delta}_{i}\right)\left(-\dot{\breve{\delta}}_{1}\right) / \eta_{i} \\
\leq & -\tilde{\boldsymbol{I}}^{T} R \tilde{\boldsymbol{I}}-\tilde{\boldsymbol{I}}^{T} K_{e} \dot{\tilde{\boldsymbol{q}}}+\varsigma\|\tilde{\boldsymbol{I}}\|\|\boldsymbol{x}\| \\
\leq & -\tilde{\boldsymbol{I}}^{T} R \tilde{\boldsymbol{I}}+\left(\varsigma+\alpha_{k}\right)\|\boldsymbol{x}\|\|\tilde{\boldsymbol{I}}\|
\end{aligned}
$$


where $\alpha_{k} \triangleq\left\|K_{e}\right\|$. Based on (30) and (34), $\dot{V}$ can be expressed as

$$
\begin{aligned}
\dot{V} \leq & -\left(\gamma \lambda_{q}-3\left\|B_{d}\right\|-2 \vartheta\|\dot{\boldsymbol{q}}\|\right)\|\boldsymbol{x}\|^{2}+\beta_{1}\|\boldsymbol{x}\|\|\tilde{\boldsymbol{I}}\|-\tilde{\boldsymbol{I}}^{T} R \tilde{\boldsymbol{I}} \\
\leq & -\left(\gamma \lambda_{q}-3\left\|B_{d}\right\|-2 \vartheta\|\dot{\boldsymbol{q}}\|-\mu_{1}\right)\|\boldsymbol{x}\|^{2} \\
& -\lambda_{r}\left(\|\tilde{\boldsymbol{I}}\|-\nu_{1}\|\boldsymbol{x}\|\right)^{2} \\
\leq & -\left(\gamma \lambda_{q}-3\left\|B_{d}\right\|-2 \vartheta\|\dot{\boldsymbol{q}}\|-\mu_{1}\right)\|\boldsymbol{x}\|^{2}
\end{aligned}
$$

where

$$
\begin{aligned}
& \beta_{1} \triangleq\left(3 \alpha_{n}+\varsigma+\alpha_{k}\right), \mu_{1} \triangleq \frac{\beta_{1}^{2}}{4 \lambda_{r}} \\
& \nu_{1} \triangleq \frac{\beta_{1}}{2 \lambda_{r}}, \lambda_{r} \triangleq \inf \frac{\tilde{\boldsymbol{I}}^{T} R \tilde{\boldsymbol{I}}}{\|\tilde{\boldsymbol{I}}\|^{2}} .
\end{aligned}
$$

From the definitions of $\boldsymbol{x}$ and $\boldsymbol{x}_{v}$, it is easy to show from (28) that

$$
\|\dot{\boldsymbol{q}}\| \leq\|\boldsymbol{x}\|+\left\|\dot{\boldsymbol{q}}_{d}\right\| \leq\left\|\boldsymbol{x}_{v}\right\|+\left\|\dot{\boldsymbol{q}}_{d}\right\| \leq \sqrt{\frac{V}{\lambda_{v 1}}}+\left\|\dot{\boldsymbol{q}}_{d}\right\|
$$

which can be used to place an upper bound for $\dot{V}$ as

$$
\begin{aligned}
\dot{V} & \leq-\left(\gamma \lambda_{q}-3\left\|B_{d}\right\|-2 \vartheta\left\|\dot{\boldsymbol{q}}_{d}\right\|-2 \vartheta \sqrt{\frac{V}{\lambda_{v 1}}}\right)\|x\|^{2} \\
& \leq-\rho\|\boldsymbol{x}\|^{2} \text { for } V<\lambda_{v 1}\left(\frac{\gamma \lambda_{q}-3\left\|B_{d}\right\|-2 \vartheta\left\|\dot{\boldsymbol{q}}_{d}\right\|}{2 \vartheta}\right)^{2}
\end{aligned}
$$

where $\rho$ is a positive constant. When $V<\lambda_{v 1}\left(\left(\gamma \lambda_{q}-3\left\|B_{d}\right\|-\right.\right.$ $\left.\left.2 \vartheta\left\|\dot{\boldsymbol{q}}_{d}\right\| / 2 \vartheta\right)\right)^{2}, V$ is positive definite and $\dot{V}$ is negative semi-definite, we have $V(0) \geq V$ for all $t \geq 0$. From (28), we have $V(0) \leq$ $\lambda_{v 2}\left\|\boldsymbol{x}_{v}(0)\right\|^{2}$ which allows (37) to be written as

$$
\begin{aligned}
\dot{V} & \leq-\rho\|\boldsymbol{x}\|^{2} \text { for } \quad \lambda_{v 2}\left\|\boldsymbol{x}_{v}(0)\right\|^{2} \\
& <\lambda_{1}\left(\frac{\gamma \lambda_{q}-3\left\|B_{d}\right\|-2 \vartheta\left\|\dot{\boldsymbol{q}}_{d}\right\|}{2 \vartheta}\right)^{2}
\end{aligned}
$$

which yields the gain condition of (21).

To complete the proof, it is necessary to show that $q \rightarrow 0$ as $t \rightarrow \infty$. Since $\dot{V}$ is negative semi-definite, $x, \tilde{\alpha}_{a k}$, and $\tilde{I}$ are all bounded, which implies that all signals on the right side of (23) are bounded. The boundedness of $\boldsymbol{x}$ implies that $\boldsymbol{x}$ is uniformly continuous. Also, form (38), we can show that $\boldsymbol{x} \in \mathcal{L}_{2}^{3 n}$. Therefore, as a direct consequence of Barbalat's lemma, we have $\lim _{t \rightarrow \infty} \boldsymbol{x}=$ 0 , which implies the result given in Theorem.

Remark: The stability result is semi-global since the gain $\gamma$ can be arbitrarily increased to encompass any set of initial conditions to provide for asymptotic link position tracking. However, we should mention that too large $\gamma$ may lead to a high gain scheme. Therefore, a trade-off should be made between the control accuracy and control gain.

\section{A Simulation Example}

As an illustration, we will apply the adaptive algorithm (17)-(20) to a two-link robot arm with DC actuators as used in [16]. The detailed description of robot model may refer to [16].

The desired $\boldsymbol{I}_{d}$ is synthesized by (6) where $\kappa=8, \gamma^{2}=30, \Gamma=$ $15 I$, and $\sigma=0.2$. The initial values of $\hat{\alpha}_{a k}$ are chosen as $\hat{\alpha}_{a k}(0)=$ $[1.0,0.35,0.19,0.1,2.2,2.3,1.2,0.4,0.2,0.12,2.5,2.6]^{T}$. In the projection operator, $\theta_{i \min }$ is chosen as $\theta=[0,0,0,0,0,0,0,0,0$, $0,0,0]^{T}$ and $\theta_{i \max }$ is chosen as $\theta=[3,2,1,1,5,5,3,2,1$, $1,7,7]^{T}$. The controller is then synthesized by (17) where $\eta_{1}=$

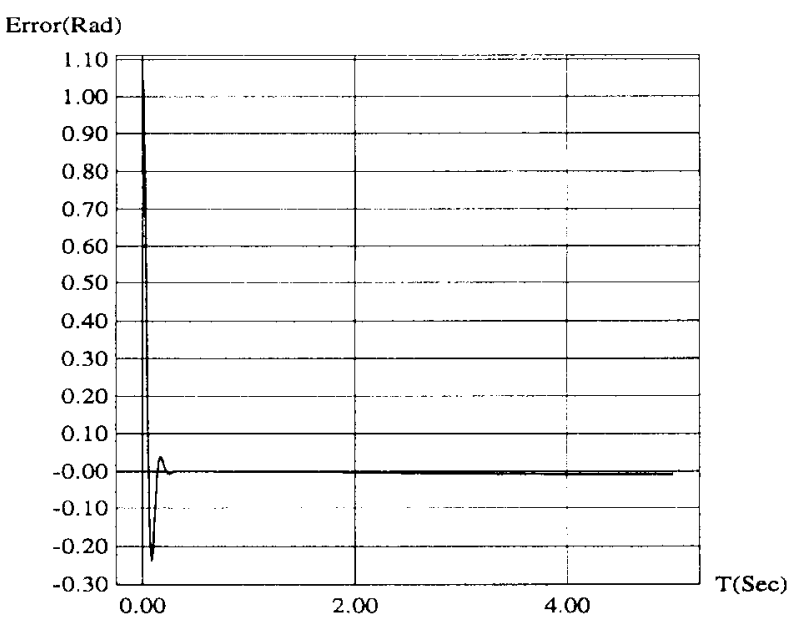

Fig. 1. Tracking error of joint 1 .

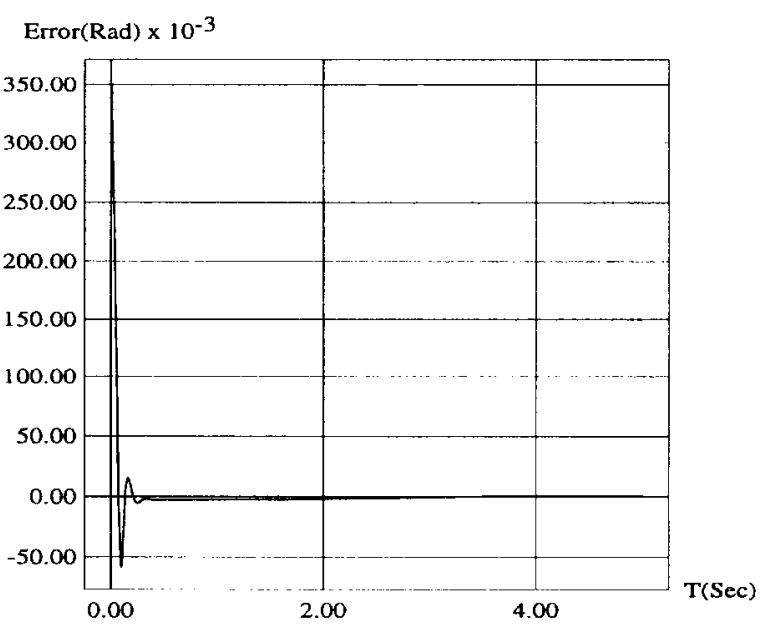

Fig. 2. Tracking error of joint 2 .

$1 \times 10^{-11}, \eta_{2}=1 \times 10^{-6}$, and $\eta_{3}=1 \times 10^{-6}$. The initial values of $\hat{\delta}_{i}$ are chosen as $\hat{\delta}_{1}(0)=8 \times 10^{-5}, \hat{\delta}_{2}(0)=1$, and $\hat{\delta}_{3}(0)=5$.

Choosing the same desired trajectories and initial displacements and velocities as in [16], the results of the simulation are shown in Figs. 1 and 2. Fig. 1 shows the trajectory tracking error of joint 1. Fig. 2 shows the trajectory tracking error of joint 2 . Validity of this adaptive controller is confirmed for the purpose of trajectory tracking in the presence of actuator dynamics. We should mention that selection of controller parameters can affect the system performance. For example, in the case of small $\eta_{1}, \eta_{2}$, and $\eta_{3}$, due to the slow rate of parameter adaptation, the parameter adaptation is not quick enough to arrive at a value satisfying the stability condition. Hence large values of $\eta_{1}, \eta_{2}$, and $\eta_{3}$ may improve the system response. On the other hand, too large $\eta_{1}, \eta_{2}$, and $\eta_{3}$ may cause high control gains. Unfortunately, there is no systematic approach for the selection of these values. They must be chosen using iterative simulations, and a trade-off between system response and control gains should be made.

\section{CONCLUSION}

In this paper, a hybrid adaptive control law has been proposed for rigid-link electrically-driven robot manipulators. The proposed controller is a modification of the controller in [16]. Two major limitations of the scheme in [16] have been eliminated. This implies 
that the applicability of the scheme in [16] can be greatly broadened. Simulation results verified the validity of the algorithm.

\section{ACKNOWLEDGMENT}

The authors wish to thank the referees for the precise comments and corrections that have enabled the authors to produce the final form of this paper.

\section{REFERENCES}

[1] M. C. Good, L. M. Sweet, and K. L. Strobel, "Dynamic models for control system design of integrated robot and drive systems," J. Dyn. Syst., Meas., Contr., vol. 107, pp. 53-59, 1985.

[2] R. W. Beekmann and K. Y. Lee, "Nonlinear robotic control including drive motor interactions," in Proc. Amer. Contr. Conf., Atlanta, GA, 1988, pp. 1333-1338.

[3] D. Taylor, "Composite control of direct-drive robots," in Proc. 28th Conf. Dec. Contr., 1989, pp. 1670-1675.

[4] T. J. Tarn, A. K. Bejczy, X. Yun, and Z. Li, "Effect of motor dynamics on nonlinear feedback robot arm control," IEEE Trans. Robot. Automat., vol. 7, pp. 114-122, 1991

[5] C.-Y. Su, T. P. Leung, and Y. Stepanenko, "Real-time implementation of regressor based sliding mode control scheme for robot manipulators," IEEE Trans. Ind. Electron., vol. 40, pp. 71-79, 1993.

[6] D. M. Dawson, Z. Qu, and J. J. Carrol, "Tracking control of rigidlink electrically-driven robot manipulator," Int. J. Contr., vol. 56, pp. 911-1006, 1992.

[7] M. Mahmoud, "Robust control of robot arms including motor dynamics," Int. J. Contr., vol. 58, pp. 853-873, 1993.
[8] G. Liu and A. A. Goldenberg, "Robust control of robot manipulators incorporating motor dynamics," in Proc. 1993 IEEE/RSJ Int. Conf. Intell. Robot Syst., Yokohama, Japan, 1993, pp. 68-75.

[9] C.-Y. Su and Y. Stepanenko, "On the robust control of robot manipulators including actuator dynamics," J. Robot. Syst., vol. 13, pp. 1-10, 1996.

[10] M. M. Bridges, D. M. Dawson, and X. Gao, "Adaptive control of rigidlink electrically driven robots," in Proc. IEEE Conf. Dec. Contr., 1993, pp. $159-165$

[11] S. S. Ge and I. Postlethwaite, "Nonlinear adaptive control of robots including motor dynamics," in Proc. Amer. Contr. Conf., San Francisco, CA, 1993, pp. 1423-1427.

[12] J. Yuan, "Adaptive control of robotic manipulators including motor dynamics," IEEE Trans. Robot. Automat., vol. 11, pp. 612-617, 1995.

[13] R. Colbaugh and K. Glass, "Adaptive regulation of rigid-link electrically-driven manipulators," in Proc. IEEE Int. Conf. Robot. Automat., 1995.

[14] R. Guenther and L. Hsu, "Variable structure adaptive cascade control of rigid-link electrically-driven robot manipulators," in Proc. IEEE Conf. Dec. Contr., San Antonio, TX, 1993, pp. 2137-2142.

[15] Y. Stepanenko and C.-Y. Su, "Adaptive motion control of rigid-link electrically-driven robot manipulators," in Proc. IEEE Int. Conf. Robot. Automat., 1994, pp. 630-635.

[16] C.-Y. Su and Y. Stepanenko, "Hybrid adaptive/robust motion control of rigid-link Electrically-driven robot manipulators," IEEE Trans. Robot. Automat., vol. 11, pp. 426-432, 1995.

[17] R. Klafterm, T. Chmielewski, and M. Negin, Robotic Engineering: An Integrated Approach. Englewood Cliffs, NJ: Prentice-Hall, 1989.

[18] T. Burg, D. Dawson, J. Hu, and M. de Queiroz, "An adaptive partial state-feedback controller for RLED robot manipulators," IEEE Trans. Automat. Contr., vol. 41, pp. 1024-1030, 1996.

[19] M. Krstic, I. Kanellakopoulos, and P. V. Kokotovic, Nonlinear and Adaptive Control Design. New York: Wiley, 1995. 